\title{
FOLLOW-ME: ANÁLISE DOS CONSTRUTOS QUE INFLUENCIAM NA TOMADA DE DECISÃO DE SEGUIDORES LIGADOS A INFLUENCIADORES DIGITAIS NO CONTEXTO VIRTUAL
}

\author{
Igor de Jesus Lobato Pompeu Gammaranoํㅜㄹ Everaldo Marcelo Souza da Costa, Sérgio Castro Gomes, Emilio Jose \\ Montero Arruda Filho \\ Universidade da Amazônia - UNAMA, Belém, Pará (Brasil)
}

\section{DETALHES DO ARTIGO}

\section{Histórico do Artigo:}

Recebido: 01 de outubro de 2019

Aceito: 30 de março de 2020

Disponível online: 01 de maio de 2020

Sistema de revisão "Double blind review"

Editor Científico

Ilan Avrichir

\section{Palavras-chaves:}

Influenciadores Digitais

Tomada de Decisão

Boca-a-Boca Eletrônico

\begin{abstract}
RESUMO
Objetivo: O presente estudo tem como objetivo analisar a relação existente entre os construtos Confiança, Boca-a-Boca Eletrônico (E-WOM) e Engajamento com a Tomada de Decisão de consumo dos indivíduos que seguem os Influenciadores Digitais no contexto virtual.

Método: O estudo foi desenvolvido a partir de uma abordagem quantitativa exploratória utilizando o Survey com 147 respondentes, onde foi utilizada a técnica da análise fatorial exploratória (AFE), seguido de regressão linear multivariada. Principais Resultados: Os resultados apontam que os construtos Confiança e Engajamento influenciam na Tomada de Decisão de consumo dos seguidores de influenciadores digitais, enquanto Boca-a-Boca Eletrônico (E-WOM) não apresentou significância na relação com a Tomada de Decisão dos indivíduos que seguem os influenciadores digitais.

Relevância/Originalidade: Embora confiança, engajamento e Boca-Boca sejam, construtos amplamente testados no marketing quanto ao comportamento de consumo, ainda não se tem evidências de como esses construtos efetivamente são percebidos e se relacionam no contexto dos influenciadores digitais. Portanto, busca-se contribuir com a literatura quanto aos efeitos desses construtos em um ambiente específico do marketing digital, significativamente novo que pode ter efeito no processo de tomada de decisão dos consumidores.

Contribuições teóricas/metodológicas: Destaca-se a utilização da AFE como necessário à verificação da existência dos construtos em um novo ambiente de geração de consumo e sua associação com a técnica de regressão multivariada para determinação dos efeitos quanto a tomada de decisão dos consumidores.
\end{abstract}

\section{Introdução}

Durante décadas, o marketing usou figuras públicas e pessoas socialmente distintas como fontes de comunicação de alta credibilidade, e estratégia para promover marcas e produtos (Dholakia \& Sternthal, 1977; Sternthal, Dholakia \& Leavitt, 1978). A intenção é influenciar as decisões dos consumidores através de canais de comunicações tradicionais, como TV e rádio, além de publicidade exterior. Com a crescente popularidade e uso de tecnologias digitais, a ascensão da Web 2.0 e o alcance global das mídias sociais em rede, as empresas adaptaram suas estratégias de marketing a esse novo e atual contexto tecnológico digital em constante mudança (Coleman, Jain, Bahnan \& Chene, 2019; Usman \& Okafor, 2019).
Devido a esses novos canais de comunicação, consumidores podem ter acesso a mais informações sobre um dado produto, podendo inclusive, compará-lo com produtos similares em características de usabilidade e preço (Usman \& Okafor, 2019). Esse aspecto exige que a fonte de comunicação seja confiável aos consumidores para que a informação seja compreendida e aceita em sua totalidade, como estratégia realmente eficiente para o sucesso do relacionamento entre emissor e receptor (Martensen, Brockenhuus-Schack, \& Zahid, 2018).

Nesse sentido, as fontes de comunicação que buscam informar seus receptores de maneira clara e eficiente, gozam de maior confiança e reputação (Yahia, Al-Neama \& Kerbache, 2018). Celebridades 
como, o jogador de futebol Cristiano Ronaldo, com mais de 160 milhões de seguidores e a cantora Ariana Grande, com 150 milhões, só na rede social Instagram, comprovam que suas habilidades e credibilidade são diferenciais, transmitindo assim a confiança necessária à geradora de engajamento das pessoas com suas imagens (Passari \& Kumar, 2017).

Esse processo ocorre pelo vínculo emocional gerado a partir da qualidade das informações transmitidas. Assim, os seguidores da fonte informante passam a ter o sentimento de inclusão ao grupo social, que no caso das redes sociais digitais, pode ser definido como pertencimento digital (Scheinbaum, 2016). Esse sentimento é fundamental à geração de engajamento nas redes sociais (Passari \& Kumar, 2017).

Esses processos emocionais podem gerar a disposição do seguidor para o "Boca a Boca" eletrônico, o que significa que seguidores podem querer falar bem ou mal da fonte da informação (Lin, Bruning \& Swarna, 2018). No caso das redes sociais, esse processo está relacionado a disposição de compartilhar e replicar as informações gerada pelos influenciadores digitais (Chatzigeorgiou, 2017).

De forma natural e autêntica os influenciadores digitais promovem comportamentos e criam novos padrões de consumo, dialogando diretamente com seus seguidores e aprimorando a conveniência da marca de uma maneira sutil e menos invasiva. Dependendo do grau de engajamento dos seguidores, esses padrões de influência digital podem produzir resultados reais para as empresas e suas marcas, variando de maior difusão e consumo dessas por aqueles que acompanham os influenciadores digitais (Jiménez-Castillo \& Sánchez-Fernández, 2019)

Assim, baseado nos fenômenos relacionados ao impacto dos influenciadores digitais no comportamento do consumidor através da influência e persuasão social e emocional (Jiménez-Castillo \& Sánchez-Fernández, 2019), este estudo busca responder a seguinte questão: qual a relação dos construtos Confiança, Boca-a-Boca Eletrônico e Engajamento com a Tomada de Decisão dos indivíduos que seguem os Influenciadores Digitais?

Portanto, o objetivo do trabalho é verificar a relação da confiança, Boca-a-Boca eletrônico e engajamento na tomada de decisão de consumo dos seguidores de influenciadores digitais. Esse estudo se justifica pela necessidade exploratória de verificação de como esses construtos podem gerar vínculos entre seguidores e influenciadores digitais. Além disso pode explicar como o vínculo emocional afeta a decisão de consumo de seguidores em relação a marcas endossadas pelos influenciadores digitais.

Outro aspecto relevante como possível contribuição é como a liderança de opinião dos influenciadores digitais pode, dentro do cenário de consumo de diversos produtos, estar sendo percebida pelos seguidores. Deste modo, avaliar os efeitos da Confiança (Hwang \& Kim, 2007), Boca-aBoca Eletrônico (E-WOM) (Chatzigeorgiou, 2017) e Engajamento (Kumar et al., 2010) pode ser diferencial na elaboração de estratégias de marketing das organizações que utilizam influenciadores digitais para gerar tomada de decisão a favor de seus produtos (Djafarova \& Rushworth, 2017).

O estudo foi desenvolvido a partir de uma abordagem quantitativa exploratória utilizando o survey como método de coleta de dados, sendo a técnica de análise fatorial exploratória (AFE) necessária à avaliação dos construtos no contexto do marketing digital, esse processo é seguido de uma análise de regressão com o objetivo de identificar a relação entre variáveis independentes (confiança, Boca-a-Boca eletrônico e engajamento) com a variável dependente (tomada de decisão).

A literatura indica algumas características expressas pelos influenciadores digitais que envolvem emocionalmente sua rede de seguidores, dentre as quais confiança e engajamento aparecem como determinantes na ampliação do número de seguidores, bem como do sucesso dos influenciares digital (Hwang \& Kim, 2007; Kumar et al., 2010). Porém, pouco estudos como os de Jiménez-Castillo e Sánchez-Fernández (2019) e Dahka, Hajiheydari e Rouhani (2020) tem envolvido o Boca-a-Boca eletrônico como um construto que pode explicar o envolvimento dos seguidores com seus influenciadores. Nesse sentido, esse estudo busca avançar quanto ao nível de influência dessa variável na tomada de decisão de consumo dos seguidores de influenciadores digitais. O artigo divide-se da seguinte forma: após esta introdução a qual apresenta os objetivos e desenvolvimento do estudo, a segunda seção descreve o referencial teórico que concedeu base para a definição dos construtos, a partir da compreensão de significados, atitudes, comportamentos subjetivos e intenções que 
Follow-Me: Análise dos Construtos que Influenciam na Tomada de Decisão de Seguidores Ligados a Influenciadores Digitais no Contexto Virtual

conectam emocionalmente os Influenciadores Digitais aos seus Seguidores. A seguir, a metodologia que sistematiza a coleta e análise dos dados trabalhados é delineada. Por fim, são abordados os resultados e as considerações finais do estudo.

\section{Referencial Teórico}

\section{Confiança}

A confiança se apresenta como um dos construtos que concede a estabilidade e a segurança psicológica que os indivíduos precisam para absorverem informações e executarem comportamentos (Martensen, Brockenhuus-Schack, \& Zahid, 2018). Logo, a confiança pode ser definida como uma crença de que a outra parte no intercâmbio social agirá de forma ética e socialmente adequada, não agindo de forma oportunista (Hwang \& Kim, 2007).

A confiança se estabelece quando uma das partes confia na credibilidade, habilidade, conhecimento, reputação (Yahia, Al-Neama \& Kerbache, 2018), integridade e benevolência dos demais indivíduos com os quais se relaciona (Gefen \& Straub, 2004). Percebe-se que fatores como experiência com o produto e conhecimento, reputação, hedonismo (Yahia, Al-Neama \& Kerbache, 2018), utilidade, segurança, qualidade (Kim \& Peterson, 2017) e expertise (Thakur et al., 2016), também são fatores que possibilitam aos usuários de redes sociais confiarem nas opiniões, sugestões e indicações dos Influenciadores Digitais, que esses seguem. A formação da confiança também se baseia na relação de dependência na perícia, conhecimento e knowhow dos Influenciadores Digitais que manuseiam ou indicam produtos ou serviços (Johnson \& Grayson, 2005).

A confiança se mostra como um construto com características unidimensionais que permite avaliar relações como a dos influenciadores com os seus seguidores (Larzelere \& Huston,1980; Martensen, Brockenhuus-Schack \& Zahid, 2018) e engajá-los (Wongkitrungrueng \& Assarut, 2018), haja vista que dependendo do nível de confiança e envolvimento estabelecido, um seguidor pode se tornar um defensor de seu influenciador (Sashi, 2012).

\section{Engajamento}

O engajamento é um dos fatores chave no processo de decisão de consumo dos seguidores. Pois, nota-se que quanto mais engajado o seguidor se mostra, mais propício ele estará a acatar as sugestões de seus influenciadores (Scheinbaum, 2016). Conforme Passari \& Kumar (2017), o engajamento surge quando em um relacionamento existe o sentimento de satisfação e há um forte vínculo emocional envolvido entre as partes. O engajamento também pode ser observado como uma forma de criar a interação e a participação de um determinado indivíduo em um contexto específico (Kumar et al., 2010).

Em alguns casos, o nível de engajamento pode se aprofundar devido a afetividade construída pelos seguidores, podendo chegar a expressar aspectos devocionais (Sashi, 2012). Assim, o nível de envolvimento emocional que possibilita o engajamento (Passari \& Kumar, 2017) pode ser um fator determinante para que os seguidores venham defender de maneira dogmática, as opiniões e atitudes de seus Influenciadores Digitais.

O engajamento digital pode ser otimizado pelo Influenciador Digital a partir da produção de conteúdo personalizado, visando um público específico que se sinta atendido a partir da produção de um conjunto de informações direcionadas, como se fossem projetadas tendo essas pessoas em mente (Scheinbaum, 2016). Ademais, outro fator que potencializa o engajamento digital é o pertencimento digital, que se define como o sentimento de integração de um indivíduo a uma comunidade ou grupo que possui características compatíveis com a identidade social deste usuário (Scheinbaum, 2016).

Entende-se também que o nível de engajamento dos seguidores será determinante para que esses se envolvam com as atividades ligadas aos Influenciadores Digitais no ambiente virtual. Isso possibilita que determinados seguidores possam orientar as suas decisões de consumo a partir das avaliações dos seguidores engajados, por meio do boca-a-boca eletrônico (Chu, \& Kim, 2011). Dessa forma, o engajamento surge como um construto de características unidimensionais que permitem sua mensuração com a tomada de decisão dos seguidores, quanto à compra de produtos endossados pelos influenciadores digitais (Hollebeek, Glynn \& Brodie, 2014).

Kumar et al. (2010), indica que o engajamento dos clientes por meio do boca-a-boca eletrônico, possibilita a aquisição e retenção de novos clientes e o compartilhamento de informações sobre estes indivíduos. 


\section{Boca A Boca Eletrônico (E-Wom)}

O boca-a-boca eletrônico (também conhecido como e-word-of-mouth ou E-WOM) se define como $o$ ato de influenciar e envolver pessoas no ambiente digital, por meio de declarações positivas ou negativas, a fim de que essas compartilhem mensagens com clientes potenciais inseridos neste mesmo contexto eletrônico (Chatzigeorgiou, 2017).

Conforme Shi e Wojnicki (2014), os Influenciadores Digitais, são uma das principais fontes do boca-a-boca eletrônico. Os Influenciadores Digitais também possuem o poder de persuadir sua rede de seguidores, com o intuito de influenciar suas atitudes e comportamentos (Nunes et al., 2018), estimulando a execução de ações conforme suas orientações com uma determinada frequência (Rogers, 1983). Logo, os seguidores buscam desenvolver formas e estratégias para diminuir os riscos e incertezas relacionados a tomada de decisão (Leal, Hor-Meyll, \& Pessoa, 2014).

Deste modo, informações e opiniões são transmitidas no ambiente digital por meio de vídeos, imagens, textos, ou caracteres que carregam elementos emocionais como emoticons ou emojis, que podem alcançar milhares ou até mesmo milhões de potências consumidores (Lin, Bruning \& Swarna, 2018). Sendo assim, percebe-se que um dos motivos que instiga os seguidores a comentarem positivamente ou negativamente no ambiente virtual é o desejo de controlar ou influenciar indiretamente os negócios, por meio de críticas direcionadas a futuros clientes em potencial, sobre as indicações e comportamentos dos Influenciadores Digitais (Pan \& Crotts, 2012).

A literatura também aponta que elementos como confiança, força do laço, homofilia, e influência interpessoal informal são fatores importante que influenciam na formação do boca-a-boca eletrônico (Chu \& Kim, 2011). Neste sentido, verifica-se que consumidores que apresentam experiência limitada, envolvimento emocional em relação a decisão de compra ou que percebem elevado risco na tomada de decisão, possuem uma maior propensão para buscar informações e opiniões de indivíduos semelhantes, a fim de se aconselharem sobre produtos ou marcas (Nhlabathi, 2016). Em vista disso, a confiança se apresenta como fator chave para que o boca-a-boca eletrônico se desenvolva, haja vista que é necessário estabelecer um vínculo de confiabilidade entre indivíduos para que conselhos, opiniões ou sugestão sobre produtos possam ser acatadas ao invés de buscarem informações em outras fontes (Habibi, Laroche \& Richard, 2014). Logo, consumidores menos experientes buscam conselhos de outros consumidores experientes com o intuito de resolver problemas, findar incertezas e obterem sugestões e direcionamentos que influenciarão em suas tomadas de decisões de consumo (Nhlabathi, 2016).

Seguindo essas relações, o E-WOM mesmo sendo um construto bidimensional (dimensões positivas e negativas) tem suas escolhas fortemente pautadas nas avaliações positivas, comparado com as negativas (Goyette, Ricard, Bergeron \& Marticotte, 2010). Nesse sentido a tomada de decisão pro-consumo é diretamente afetada, no ambiente virtual, pelo poder de comunicação positiva dos influenciadores digitais (Coleman et al. 2019).

\section{Tomada De Decisão}

A literatura aponta que os influenciadores digitais buscam elevar a confiança (Martensen, BrockenhuusSchack, \& Zahid, 2018) estimular o engajamento (Nunes et al., 2018) e o boca a boca eletrônico (Djafarova \& Rushworth, 2017), com o intuito de persuadir e influenciar seus seguidores em suas tomadas de decisão de consumo (Djafarova \& Rushworth, 2017).

Desse modo, os seguidores podem se mostrar satisfeitos, após serem persuadidos por seus influenciadores e tomarem a decisão por seguir uma sugestão de comportamento ou consumir uma determinada marca, produto ou serviço, uma vez que a indicação do influenciador, atendeu a expectativa do seguidor (Oliver, 1997). Portanto, estes seguidores satisfeitos poderão se manifestar por intermédio do boca a boca eletrônico, com o intuito de recompensar a empresa e serem altruístas (HennigThurau et al., 2004) e úteis para outros consumidores, auxiliando outros consumidores em suas tomadas de decisões, a partir da postagem de críticas positivas formais ou informais, sobre a experiência de consumo concretizada (Pan \& Crotts, 2012). Esta comunicação fomenta nos seguidores sentimentos de eficácia, prazer e satisfação em fornecer tais conselhos a outros (Pierce, Kostova \& Dirks, 2003).

Assim os indivíduos que executam o boca-boca eletrônico, buscam informar outros consumidores sobre produtos potenciais produtos, com o intuito de incentivar o consumo a partir de suas boas 
Follow-Me: Análise dos Construtos que Influenciam na Tomada de Decisão de Seguidores Ligados a Influenciadores Digitais no Contexto Virtual

experiências, almejando ajudar estas pessoas a tomarem decisões assertivas durante seu processo de consumo por meio da propagação de suas experiências positivas (Liu et al., 2018). Desta forma, a tomada de decisão se apresenta como um construto unidimensional que possibilita avaliar o nível de consciência quanto as possibilidades existentes e concluir sobre o melhor caminho a seguir (Bennett et al. 2010).

\section{Metodologia}

Devido à natureza do fenômeno estudado, que envolve a necessidade exploratória das relações no campo de atuação dos influenciadores digitais, o método de coleta de dados escolhido foi um Survey (Hair et al., 2005, p.151-173), pois os valores percebidos pelos seguidores são melhor capitados com dados primários, gerados a partir da criação dos cenários específicos à pesquisa. Dentro do método, foi assumido o modelo auto administrável pelos entrevistados.

Com isso, a unidade de análise, indivíduos que acompanham os conteúdos transmitidos por influenciadores digitais no ambiente das redes sociais virtuais, ficam isoladas da influência do pesquisador, o que assegura a isonomia de percepção e desejo dos respondentes (Malhotra et al., 2005, p.257-277). Desta forma, o método de survey eletrônico apresenta mais vantagens do que desvantagens, comparado com outros métodos de coletas de respostas administrado pelo próprio respondente (Evans \& Mathur, 2005). Devido a isso, o questionário foi postado na plataforma SurveyMonkey.com

no período de 03/03/2018 a 10/07/2018 e compartilhado com potenciais respondentes nas redes sociais (WhatsApp, Instagram e Facebook).

Esse procedimento ocorreu a partir da criação de um cenário onde foi solicitado aos respondentes que pensassem em um influenciar digital e na rede social de sua preferência. Além disso, foi feita uma pergunta filtro com característica Dami (sim ou não); sendo: "Você costuma seguir influenciadores digitais no ambiente virtual?". Quando a resposta foi "não", automaticamente o questionário foi descartado da amostra (Malhotra et al., 2005). Esse procedimento visou garantir que apenas respondentes inseridos no cenário de seguidores de influenciadores digitais participassem da pesquisa.

Desta forma, essa pesquisa teve carácter não probalístico (Malhotra et al., 2005) sendo que o recorte amostral visa demonstrar a existência de efeitos dos construtos independentes (confiança, engajamento e E-WON positivo) no construto dependente (Tomada de decisão). Os itens formadores desses construtos foram adaptados dos estudos apresentados na tabela 1.

Tabela 1: Apresentação das escalas dos construtos propostos.

\begin{tabular}{|l|l|}
\hline CONSTRUTOS & AUTORES \\
\hline Confiança & Larzelere \& Huston (1980) \\
\hline E-Wom & Goyette et al. (2010) \\
\hline Engajamento & Hollebeek et al. (2014) \\
\hline Tomada de Descisão & Bennett et al. (2010) \\
\hline
\end{tabular}

Fonte: Elaboração Própria (2019)

Foram enviados 530 convites para participação na pesquisa, com o critério de serem pessoas acima de 18 anos que apresentavam perfil ativo nas redes sociais e que admirassem alguma personalidade na internet, porém apenas 147 respondentes retornaram a pesquisa de forma completa. Essa taxa de respondentes $(27,7 \%)$ aproximadamente, embora acima das taxas para estudos de questionário auto administrável (Evans \& Mathur, 2005), reforça a característica da conveniência dos respondentes e da impossibilidade de generalização dos resultados (Malhotra et al., 2005, p.257-277).
O planejamento da pesquisa levou em conta a validade substantiva dos relacionamentos teóricos propostos (Pasquali, 2007). Nesse sentido, o questionário manteve as características fundamentais das escalas; porém apenas foram adotadas medidas estatísticas padronizadas em escala contínua de 7 pontos, sendo 1 - discordo totalmente e 7 - concordo totalmente. Quanto aos procedimentos de pesquisa, foi utilizado a análise fatorial exploratória (AFE), essa técnica deve ocorrer em pesquisas exploratórias pela necessidade de se produzir informações sobre características latentes presentes em um conjunto de variáveis que formam 
um construto inicial (Bido, 2018). Para o desenvolvimento desse tipo de análise é necessário a verificação dos pressupostos da unidimensionalidade e viés do método (Hair et al., 2014). No contexto do presente estudo, essa técnica pode ser utilizada com parcimônia, devido a inexistência de escalas específicas dos construtos envolvidos para o cenário inovador para tomada de decisão de seguidores de influenciadores digitais (Bido, 2018).

A unidimensionalidade visa avaliar a adequação dos dados amostrais ao uso da AFE, ou seja, utilizouse o teste Kaiser-Meyer-Olkin (KMO), para avaliar se a amostra tem distribuição adequada para análise fatorial. Sendo assim, a hipótese a ser testada é que a matriz de variância/covariância não é uma matriz identidade (Lorenzo-Seva; Timmerman; Kiers, 2011).

Já o viés do método ocorre devido o modelo de coleta de dados utilizado na pesquisa, ou seja, o formato da coleta com os dados obtidos a partir de survey, em que as variáveis exógenas e endógenas são coletadas de uma única fonte (mesmo respondente, mesmo formato de respostas, mesma forma de coleta e no mesmo instante), pode ocasionar o common method bias, que constata viés dos coeficientes, ou seja, relação entre as variáveis latentes (HAIR et al., 2014).

Para solução desse problema adotar-se-á o teste de Harman, que é o mais utilizado, considerando a Análise Fatorial Exploratória (AFE) com todos os itens para uma mesma análise através do método de componentes principais (Bido, 2018). Os indicadores levantados e analisados através de AFE se sustentam nos estudos de Hair et al. (2010), assim como todas as medições foram realizadas e consolidadas no software IBM SPSS Statistics, versão 23, conforme resultados apresentados a seguir.

Tabela 2 - Teste do poder amostral

\begin{tabular}{lll}
\hline \multirow{3}{*}{ Input } & \multicolumn{1}{c}{ Post hoc: Compute achieved power } \\
& $=$ One
\end{tabular}

Fonte: Dados da amostra com o uso do G*Power 3.1

A qualidade da amostra foi avaliada pelo software G*Power 3.1 para realizar a verificação do poder amostral (Faul et al., 2007). Para esse teste de verificação amostral foi realizado a rotina baseada em teste $\mathrm{t}$ com análise post hoc, pois os dados já eram conhecidos (ver Tabela 2). Neste caso, o poder estatístico encontrado foi de aproximadamente $98,6 \%$, assumindo-se um efeito médio de 0,15, totalmente adequado para a pesquisa.

\section{Análise Dos Dados}

O banco de dados foi tabulado utilizando o SPSS 23. Após a tabulação, foram realizadas análises descritivas para verificar o perfil amostral e comportamento exploratório das características observadas nos construtos descritos na literatura como importantes para o consumo.

\subsection{Perfil Amostral e Análise Fatorial Exploratória (AFE).}

Conforme observado na Tabela 03, a amostra do estudo teve predominância da participação masculina e jovem. Isto porque, conforme Dunkley (2017), grande parte dos indivíduos que seguem influenciadores digitais e buscam obter informações sobre produtos e marcas no ambiente digital, são pertencentes a geração $Z$, classificada como sendo a geração de indivíduos que nasceram a partir de 2001. 
Tabela 03 - Descrição dos participantes da amostra

\begin{tabular}{c|c|c|c}
\hline \multicolumn{1}{c}{ Sexo } & $\%$ & \multicolumn{1}{c}{ Idade } & $\%$ \\
\hline Masculino & 63,89 & Até 25 anos & 67,81 \\
\hline Feminino & 36,11 & 26 a 30 anos & 16,44 \\
\hline & & 31 a 35 anos & 4,79 \\
\hline & & 36 a 40 anos & 4,79 \\
\hline & 41 a 45 anos & 5,48 \\
\hline & $\begin{array}{c}\text { Mais de } 45 \\
\text { anos }\end{array}$ & 0,68 \\
\hline
\end{tabular}

Fonte: Elaboração Própria (2019)

Feita uma primeira rodada de avaliação dos itens dos construtos envolvidos, com vistas a unidimensionalidade, o primeiro teste a ser analisado é o KMO, também conhecido como índice de adequação da amostra. O KMO é definido como um índice estatístico que indica a proporção de variância dos itens que podem ser explicados por uma variável latente (Lorenzo-Seva, Timmerman \& Kiers, 2011). Este teste, indica a adequação e a aplicação da AFE para o conjunto de dados coletados (Hair et al., 2005). Conforme Hutcheson e Sofroniou (1999), valores entre 0,5 e 0,7 são definidos como medianos e valores entre 0,7 e 0,8 são avaliados como bons. Logo, observa-se que os valores dos construtos Confiança, E-WOM e Engajamento estão entre o intervalo de valores considerados bons pela literatura. Contudo, a tomada de decisão apresentou um valor definido como mediano.

Portanto, os valores apresentados na Tabela 04 com a aplicação do teste de KMO indicam que a utilização da técnica de análise fatorial exploratória é adequada a amostra avaliada, possibilitando o prosseguimento da aplicação da AFE.

Tabela 04 - Teste de KMO e Bartlett

\begin{tabular}{|c|c|c|c|c|c|}
\hline \multicolumn{2}{|c|}{ Teste/Dimensões } & Confiança & E-WOM & Engajamento & $\begin{array}{c}\text { Tomada de } \\
\text { decisão }\end{array}$ \\
\hline \multicolumn{2}{|c|}{ Kaiser-Meyer-Olkin } & 0,798 & 0,747 & 0,704 & 0,672 \\
\hline $\begin{array}{c}\text { Bartlett's } \\
\text { Test of } \\
\text { Sphericity }\end{array}$ & $\begin{array}{c}\text { Approx. } \\
\text { Chi-Square }\end{array}$ & 213,00 & 189,245 & 253,798 & 104,629 \\
\cline { 2 - 6 } & df & 6 & 10 & 10 & 3 \\
\hline
\end{tabular}

Fonte: Elaboração Própria (2019)

Outro teste analisado é o teste de comunalidade que representa a validade convergente dos itens formadores dos construtos apresentados na literatura, no qual são aferidas as correlações das variáveis indicadas por intermédio dos fatores observados. Com isso, quanto maior o nível de comunalidade, mais robusta será a explicações das variáveis pelo fator. As comunalidades que estiverem acima de 0,5 são consideradas aceitáveis para os fatores analisados (Bido, 2018).
Observa-se na Tabela 05 que todos os fatores do construto Confiança e Tomada de decisão estão em intervalos considerados aceitáveis para níveis de comunalidade. Os fatores E-WOM2, E-WOM5, ENGAJ 2 e ENGAJ 3, também estão dentro dos níveis aceitáveis de comunalidade. Contudo, os fatores E-WOM 1, E-WOM 3, ENGAJ 1, ENGAJ 4 e ENGAJ 5 apresentaram baixos níveis de comunalidade. Desses últimos, foram retirados os valores com a menor carga de comunalidade, com vista a melhoria da qualidade convergente dos itens remanescentes. 
Tabela 05 - Teste de Comunalidade

\begin{tabular}{c|c|c}
\hline Construto & Itens & Comunalidades \\
\hline Confiança & Conf1 &, 547 \\
\cline { 2 - 3 } & Conf2 &, 629 \\
\cline { 2 - 3 } & Conf3 &, 801 \\
\cline { 2 - 3 } & Conf4 &, 703 \\
\hline \multirow{4}{*}{ E-WOM } & E-WOM1 &, 355 \\
\cline { 2 - 3 } & E-WOM2 &, 540 \\
\cline { 2 - 3 } & E-WOM3 &, 429 \\
\cline { 2 - 3 } & E-WOM4 &, 551 \\
\cline { 2 - 3 } & E-WOM5 &, 712 \\
\hline \multirow{4}{*}{ Engajamento } & ENGAJ1 &, 475 \\
\cline { 2 - 3 } & ENGAJ2 &, 741 \\
\cline { 2 - 3 } Tomada de & ENGAJ3 &, 760 \\
\cline { 2 - 3 } decisão & ENGAJ4 &, 342 \\
\cline { 2 - 3 } & ENGAJ5 &, 274 \\
\cline { 2 - 3 } & TD1 &, 659 \\
\cline { 2 - 3 } & TD2 &, 735 \\
\cline { 2 - 3 } & TD3 &, 609 \\
\hline
\end{tabular}

Fonte: Elaboração Própria (2019)

No teste referente a Variância Total Explicada apresentado na Tabela 06, busca-se analisar à porção de variância comum que um fator, ou um grupo de fatores, obtém a partir de um conjunto de dados específicos. Observa-se que os índices de extração dos construtos analisados estão todos acima de 50\% conforme recomendado por Chin (1998, p.321), sendo considerado aceitável para estudos que trabalham Análises Fatoriais Exploratórias. Assim, há validade convergente no nível dos fatores está preservada.

Tabela 06 - Teste do Total de Variância Explicada

\begin{tabular}{c|c}
\hline Construtos & AVE \\
\hline Confiança & $66,13 \%$ \\
\hline E-WOM & $52,18 \%$ \\
\hline Engajamento & $51,83 \%$ \\
\hline Tomada de decisão & $66,74 \%$ \\
\hline
\end{tabular}

Fonte: Elaboração Própria (2019)

Mesmo a variância total explicada e variância total explicada, apenas dos construtos apresentando resultados acima de 50\%, é que tiveram seus itens excluídos (Hair et al., 2010). necessário analisar em uma segunda rodada, os Deste modo, ao analisar o resultado do KMO itens dos construtos que tiveram baixas taxas de gerado após a segunda rodada, observa-se na comunalidade. Após a exclusão dos itens os fatores Tabela 07 que os resultados indicam uma E-WOM 1, E-WOM 3, ENGAJ 1, ENGAJ 4 e ENGAJ 5, adequação amostral à análise fatorial dos foram realizados os testes de $\mathrm{KMO}$, comunalidade construtos E-WOM e Engajamento. 
Tabela 07 -Teste KMO e Barttlet de E-WOM e Engajamento

\begin{tabular}{c|c|c|c}
\hline \multicolumn{2}{c|}{ Teste/Dimensões } & E-WOM & Engajamento \\
\hline \multicolumn{2}{c|}{ Kaiser-Meyer-Olkin } & 0,705 & 0,624 \\
\hline \multirow{2}{*}{$\begin{array}{c}\text { Bartlett's } \\
\text { Test of } \\
\text { Sphericity }\end{array}$} & $\begin{array}{c}\text { Approx. Chi- } \\
\text { Square }\end{array}$ & 103,685 & 211,130 \\
\cline { 2 - 4 } & df & 6 & 3 \\
\cline { 2 - 4 } & Sig. & 0 & 0 \\
\hline
\end{tabular}

Fonte: Elaboração Própria (2019)

Continuando a análise da segunda rodada, foi verificado na Tabela 08 a comunalidade após a retirada do item E-WOM 1 e ENGAJ 4 e ENGAJ 5. No caso específico de Engajamento, optou-se por excluir dois itens devido as cargas muito baixas obtidas na primeira rodada. Os demais fatores apresentam níveis acima de 0,5, sendo estes considerados aceitáveis pela literatura.

Tabela 08 - Análise das Comunalidades da Segunda Rodada

\begin{tabular}{c|c|c}
\hline Construto & Itens & Comunalidades \\
\hline \multirow{4}{*}{ E-WOM } & E-WOM2 & 0,563 \\
\cline { 2 - 3 } & E-WOM3 & 0,527 \\
\cline { 2 - 3 } & E-WOM4 & 0,741 \\
\cline { 2 - 3 } & E-WOM5 & 0,507 \\
\hline Engajamento & ENGAJ1 & 0,505 \\
\cline { 2 - 3 } & ENGAJ2 & 0,839 \\
\cline { 2 - 3 } & ENGAJ3 & 0,849 \\
\hline
\end{tabular}

Fonte: Elaboração Própria (2019)

Quanto a Variância Total Explicada dos construtos E-WOM e Engajamento, observa-se que seus respectivos resultados apresentaram 58,47\% (E-WOM) e 73,10\% (Engajamento), indicando uma melhor adequação a validade convergente.

Após a análise da unidimensionalidade dos construtos, foi realizado o teste para verificação do viés do método (Teste de Harman). Nesse teste, objetiva-se analisar os construtos conjuntamente para verificação da qualidade das possíveis relações advindas da literatura de marketing, comportamento do consumidor. Isso porque a observação dos itens das relações conceituais proposta tiveram fonte única de informação, isto é, mesmo respondente, mesmo formato de resposta, mesma forma de coleta e no mesmo instante.

Assim, os resultados de $\mathrm{KMO}$ do referido teste foi 0,792 , e a variância total explicada de $66,94 \%$ com a comunidade de todos os itens adequados aos critérios da validade convergente e descriminante. Desse modo, a Tabela 09 apresenta os resultados da Matriz de Componente gerada a partir do teste de Harman. 
Tabela 09 - Matriz de Componente

\begin{tabular}{ccccc}
\hline & \multicolumn{4}{c}{ Componentes } \\
\cline { 2 - 5 } Conf1 & 1 & 2 & 3 & 4 \\
\hline Conf2 &, 680 &, 082 &, 218 &, 119 \\
\hline Conf3 &, 774 &, 105 &, 218 &, 203 \\
\hline Conf4 &, 806 &, 114 &, 204 &, 105 \\
\hline E-WOM2 &, 150 &, 676 &, 225 &, 084 \\
\hline E-WOM3 &, 239 &, 660 &, 173 &, 026 \\
\hline E-WOM4 &,- 029 &, 750 &, 062 &, 074 \\
\hline E-WOM5 &, 047 &, 859 &, 074 &, 146 \\
\hline ENGAJ1 &, 152 &, 241 &, 684 &,- 014 \\
\hline ENGAJ2 &, 336 &, 190 &, 808 &, 139 \\
\hline ENGAJ &, 220 &, 116 &, 834 &, 263 \\
\hline TD1 &, 155 &, 016 &, 459 &, 678 \\
\hline TD2 &, 126 &, 101 &, 085 &, 855 \\
\hline TD3 &, 177 &, 171 &, 016 &, 775 \\
\hline Fonte: Elaborac̃o Própria & $(2019)$ & &
\end{tabular}

Fonte: Elaboração Própria (2019)

Com vistas, a verificação do impacto da confiança, engajamento e E-WOM, na tomada de decisão de consumo dos seguidores a partir de sugestões e atos sugestionáveis executados pelos influenciadores digitais. Logo, foi realizado um teste de confiabilidade, a partir dos fatores apresentados na tabela 09, resultando na Tabela 10.

\begin{tabular}{|c|c|}
\hline Construto & Alpha \\
\hline Confiança & 0,824 \\
\hline E-WOM & 0,758 \\
\hline Engajamento & 0,776 \\
\hline Tomada de decisão & 0,747 \\
\hline
\end{tabular}

A Tabela 10, apresenta valores de Alfa acima de 0,7 , o que do ponto de vista da confiabilidade dos construtos, são considerados bons (Maroco \& GarciaMarques, 2006). Esse procedimento, visa a confiabilidade dos construtos teóricos envolvidos no teste de Harman (Hair et al., 2010). Deste modo, foi realizado uma regressão linear multivariada para testar o modelo da Figura 01.

Figura 01 - Modelo de influência dos construtos da tomada de decisão

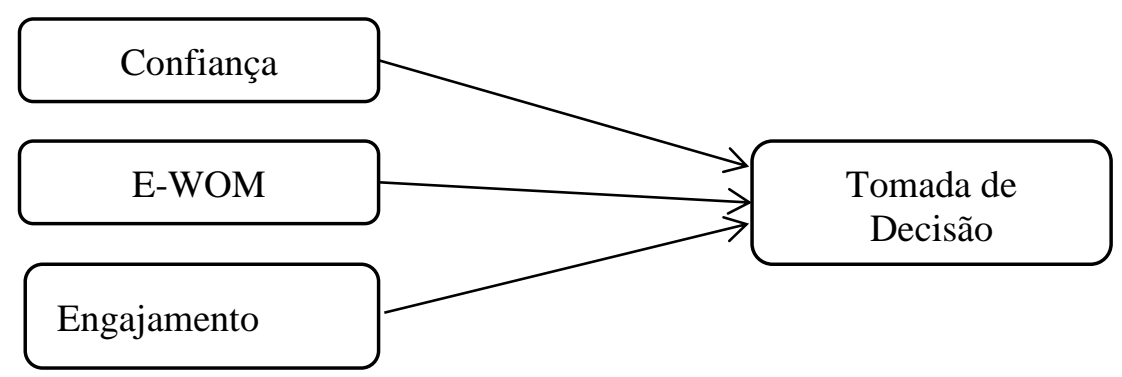

Fonte: Elaboração Própria (2019) 
Follow-Me: Análise dos Construtos que Influenciam na Tomada de Decisão de Seguidores Ligados a Influenciadores Digitais no Contexto Virtual

Assim, tendo como base o modelo teórico demonstrado na Figura 01, uma vez que os seguidores confiam nas características percebidas dos influenciadores digitais, tais como reputação, expertise, dentre outras, estes indivíduos se tornam mais propensos a acatarem as sugestões, explícitas ou implícitas, executadas pelos influenciadores, confiando nestas informações para tomarem suas decisões de consumo (Hwang \& Kim, 2007).

Além disso, os seguidores também podem servir como vetores de comunicação entre os influenciadores digitais e outros seguidores, por meio do boca-a-boca eletrônico (E-WOM) ligado ao compartilhamento de informações que também possam influenciar na tomada de decisão de consumo por terceiros (Chatzigeorgiou, 2017).
O nível de engajamento dos seguidores para com seus influenciadores digitais, também pode influenciar em suas tomadas de decisões de consumo, uma vez que os vínculos emocionais e a interação cotidiana entre estes indivíduos (Kumar et al., 2010), estabelece uma maior proximidade deixando os seguidores mais propensos a tomarem suas decisões baseadas nas sugestões compartilhadas pelos influenciadores digitais que seguem.

Devido a isso, os resultados da regressão devem demonstrar os efeitos de cada construto envolvido (variáveis independentes) na tomada de decisão (variável dependente) (ver tabela 11).

Tabela 11 - Regressão linear múltipla

\begin{tabular}{|c|c|c|c|c|c|c|c|c|c|}
\hline \multirow{2}{*}{\multicolumn{2}{|c|}{ Model }} & \multicolumn{2}{|c|}{$\begin{array}{l}\text { Unstandardized } \\
\text { Coefficients }\end{array}$} & \multirow{2}{*}{$\begin{array}{c}\text { Standardized } \\
\text { Coefficients } \\
\text { Beta }\end{array}$} & \multirow[t]{2}{*}{$t$} & \multirow[t]{2}{*}{ Sig. } & \multicolumn{2}{|c|}{$\begin{array}{l}\text { Collinearity } \\
\text { Statistics }\end{array}$} & \multirow{2}{*}{$\mathrm{R}^{2}$ Ajust } \\
\hline & & $B$ & $\begin{array}{l}\text { Std. } \\
\text { Error }\end{array}$ & & & & Tolerance & VIF & \\
\hline 1 & (Constant) & 0,690 & 0,606 & & 1,138 & 0,257 & & & \multirow{4}{*}{0,206} \\
\hline & Confiança & 0,321 & 0,113 & 0,245 & 2,838 & 0,005 & 0,731 & 1,368 & \\
\hline & E-WOM & 0,120 & 0,083 & 0,117 & 1,441 & 0,152 & 0,824 & 1,213 & \\
\hline & Engajamento & 0,277 & 0,109 & 0,229 & 2,532 & 0,012 & 0,664 & 1,506 & \\
\hline
\end{tabular}

*1. Variável dependente: Tomada de decisão

Fonte: Elaboração Própria (2019)

Conforme a Tabela 11, apenas Confiança e Engajamento deram significativos para tomada de decisão. O modelo, apresenta valores de $\mathrm{R}^{2}$ ajustado= 0,206 , o que significa um grau de explicação quanto a tomada de decisão de 20,06\%. Esse resultado é considerado aceitável pela literatura de marketing (Luigi et al., 2012).

Deste modo, a partir dos resultados obtidos, observamos que os construtos Confiança e Engajamento, influenciam na tomada de decisão de consumo dos seguidores. Tal fato ocorre, devido os seguidores perceberem provas sociais (Schaefer, 2012) compartilhadas pelos influenciadores digitais, como uma forma de legitimar suas experiências e conhecimentos sobre um determinado assunto ou produto, tornando suas recomendações mais seguras e confiáveis. Logo, a partir do compartilhamento de informações que demonstrem publicamente que os influenciadores digitais possuem experiência com o produto, habilidades, conhecimentos, credibilidade e um forte senso de reputação (Yahia, Al-Neama \& Kerbache, 2018), torna-se mais fácil e atrativo para os seguidores confiarem nesses indivíduos, adotando-os como base para suas tomadas de decisão de consumo.

O engajamento apresenta influência na tomada de decisão devido ao forte vínculo emocional e relacional construído pelos seguidores em relação aos influenciadores que seguem constantemente (Passari \& Kumar, 2017). A participação constante dos seguidores e a interação dos mesmos com os conteúdos compartilhados pelos influenciadores digitais, por meio de curtidas e comentários, intensifica a proximidade e faz com que os seguidores estejam participando e em constante contato com as recomendações feitas pelos influenciadores digitais (Kumar et al., 2010). Assim, é mais fácil seguidores serem convencidos a adotar uma sugestão do 
influenciador digital no quando interagem constantemente, e com consequente efeito na tomada de decisão.

Em relação ao boca-a-boca eletrônico, os resultados demonstram que muito embora o seguidor fale do influenciador digital que ele segue para outros indivíduos, este ato não influencia na sua tomada de decisão pessoal. Isso pode ocorrer pelo fato de os seguidores desejarem compartilhar informações e sugestões advindas de seus influenciadores como uma forma de altruísmo para com os outros ou até mesmo para observar primeiro se as sugestões compartilhadas são realmente eficazes ao que se propõe (Leal, Hor-Meyll, \& Pessoa, 2014). Logo, é possível que somente após observação criteriosa dos resultados positivos da aplicação da sugestão compartilhada pelo influenciador digital, o seguidor venha a adotar a sugestão em suas tomadas de decisões pessoais.

\section{Conclusão}

O presente artigo buscou verificar a validade substantiva de alguns construtos envolvidos no cenário dos influenciadores digitais e seus seguidores. Além disso, foi testado um modelo teórico de causalidade onde Confiança, Boca-a-Boca Eletrônico e Engajamento tem efeitos na tomada de decisão de seguidores dos influenciares digitais quanto ao consumo de produtos endossados por estes.

A validade substantiva foi confirmada, porém muitos itens (características observáveis) que se apresentam validados em outros cenários para os construtos E-WOM e engajamento, não apresentaram comunalidades satisfatórias, demonstrando que as escalas utilizadas podem não ter a qualidade necessária à compreensão mais completa dos aspectos que envolvem o fenômeno dos influenciadores digitais na decisão de consumo de seus seguidores. Porém, esse trabalho contribui demonstrando que as escalas de confiança e tomada de decisão podem ser bem exploradas para esse cenário de consumo.

A análise de regressão comprovou os efeitos da confiança e engajamento na tomada de decisão, demonstrando que esses construtos são antecedentes da tomada de decisão de seguidores digitais. Esse aspecto é fundamental para que os influenciadores digitais busquem produzir conteúdos que efetivamente gerem laços de afetividade e credibilidade com os seguidores. Ainda, para as empresas que desejam divulgar seus produtos com a utilização da imagem de influenciares digitais, é importante a verificação desses construtos para o sucesso das campanhas de marketing.

Esse estudo apresenta três limitações fundamentais. A primeira, consiste no uso de escalas que se demonstraram medianamente apropriadas para verificação dos construtos envolvidos com o fenômeno dos influenciadores digitais e que não levou em conta outros construtos que poderiam gerar maior compreensão do fenômeno, como por exemplo, o status social, reputação e a autoridade apresentada pelos influenciadores digitais; a segunda é metodológica, o tipo de amostra não probabilista que impede a não generalização dos resultados, além da técnica de conjunção da análise fatorial exploratória (AFE) seguida de regressão linear. Essa estratégia de método, pode ser substituída pela modelagem de equações estruturais com maior eficiência e qualidade nos resultados; a terceira, os resultados precisam ser vistos com cautela pois ainda é necessário aprofundar a compreensão sobre o tema tanto no ponto de vista teórico quanto gerencial.

Sugere-se para estudos futuros que estes outros construtos indicados possam ser avaliados, buscando entender qual a influência que a posse e divulgação dos mesmos pelos influenciadores digitais pode gerar na Tomada de Decisão dos seguidores. Além disso, sugere-se que a amostra possa ser ampliada e que o construto Boca-a-Boca Eletrônico (E-WOM) seja avaliado do ponto de vista que envolve os comentários dos seguidores falando sobre 0 influenciador digital, sendo esta uma abordagem diferente da que foi utilizada no presente estudo, na qual se buscou avaliar o Boca-a-Boca Eletrônico executado pelos seguidores, sobre o influenciador digital e compartilhado com terceiros.

\section{Referencias}

Bennett, C., Graham, I. D., Kristjansson, E., Kearing, S. A., Clay, K. F., \& O’Connor, A. M. (2010). Validation of a preparation for decision making scale. Patient education and counseling, 78(1), 130133.

Bido, D. S., Mantovani, D. M. N., \& Cohen, E. D. (2018). Destruição de escalas de mensuração por meio da análise fatorial exploratória nas pesquisas da 
área de produção e operações. Gest. Prod., São Carlos, 25(2), 384-397.

Chatzigeorgiou, C. (2017). Modelling the impact of social media influencers on behavioural intentions of millennials: The case of tourism in rural areas in Greece. Journal of Tourism, Heritage \& Services Marketing, 3(2), 25-29.

Chu, S., \& Kim, Y. (2011). Determinants of consumer engagement in electronic word-of-mouth (E-WOM) in social networking sites. International Journal of Advertising: The Review of Marketing Communications, 30(1), 47-75. http://dx.doi.org/10.2501/IJA-30-1-047-075.

Coleman, L. J., Jain, A., Bahnan, N., \& Chene, D. (2019). Marketing the Performing Arts: Efficacy of Web 2.0 Social Networks. Journal of Marketing Development and Competitiveness, 13(1), 23-28.

Dahka, Z. Y., Hajiheydari, N., \& Rouhani, S. (2020). User response to e-WOM in social networks: how to predict a content influence in Twitter. International Journal of Internet Marketing and Advertising, 14(1), 91-111.

Dholakia, N., \& Reyes, I. (2013). Virtuality as place and process. Journal of Marketing Management, 29(13-14), 1580-1591.

Djafarova, E., \& Rushworth, C. (2017). Exploring the credibility of online celebrities' Instagram profiles in influencing the purchase decisions of young female users. Computers in Human Behavior, 68, 1-7.

Dunkley, L. (2017). Reaching Generation Z: Harnessing the Power of Digital Influencers in Film Publicity. Journal of Promotional Communications, 5(1), 31-39.

Evans, J. R., \& Mathur, A. (2005). The value of online surveys. Internet research, 15(2), 195-219.

F. Hair Jr, J., Sarstedt, M., Hopkins, L., \& G. Kuppelwieser, V. (2014). Partial least squares structural equation modeling (PLS-SEM) An emerging tool in business research. European Business Review, 26(2), 106-121.

Faul, F., Erdfelder, E., Lang, A. G., \& Buchner, A. (2007). G* Power 3: A flexible statistical power analysis program for the social, behavioral, and biomedical sciences. Behavior research methods, 39(2), 175-191.

Gefen, D., \& Straub, D. W. (2004). Consumer trust in $\mathrm{B} 2 \mathrm{C}$ e-commerce and the importance of social presence: Experiments in e-products and e-services. Omega, 32(6), 407-424.

Goyette, I., Ricard, L., Bergeron, J., \& Marticotte, F. (2010). e-WOM Scale: word-of-mouth measurement scale for e-services context. Canadian Journal of Administrative Sciences/Revue Canadienne des Sciences de l'Administration, 27(1), 5-23.

Habibi, M. R., Laroche, M. and Richard, M. O. (2014). The roles of brand community and community engagement in building brand trust on social media. Computers in Human Behaviour, 37, 152-161.

Hair Jr, J. F., Gabriel, M. L. D. D. S., \& Patel, V. K. (2014). Modelagem de Equações Estruturais Baseada em Covariância (CB-SEM) com o AMOS: Orientações sobre a sua aplicação como uma Ferramenta de Pesquisa de Marketing. Revista Brasileira de Marketing, 13(2), 44-55.

Hair, J. F., Anderson, R. E., Tatham, R. L., \& Black, W. C. (2005). Análise multivariada de dados. 5a Edição.

Hennig-Thurau, Thorsten, Kevin P. Gwinner, Gianfranco Walsh, and Dwayne D. Gremler (2004), "Electronic Word-of-Mouth via Consumer-Opinion Platforms: What Motivates Consumers to Articulate Themselves on the Internet?" Journal of Interactive Marketing, 18 (1), 38-52.

Hollebeek, L. D., Glynn, M. S., \& Brodie, R. J. (2014). Consumer Brand Engagement in Social Media: Conceptualization, Scale Development and Validation. Journal of Interactive Marketing, 28(2), 149-165. doi:10.1016/j.intmar.2013.12.002.

Hwang, Y., \& Kim, D. J. (2007). Customer selfservice systems: The effects of perceived Web quality with service contents on enjoyment, anxiety, and etrust. Decision Support Systems, 43(3), 746-760.

Jiménez-Castillo, D., \& Sánchez-Fernández, R. (2019). The role of digital influencers in brand 
recommendation: Examining their impact on engagement, expected value and purchase intention. International Journal of Information Management, 49, 366-376.

Johnson, D., \& Grayson, K. (2005). Cognitive and affective trust in service relationships. Journal of Business Research, 58(4), 500-507.

Kim, Y., \& Peterson, R. A. (2017). A Meta-analysis of Online Trust Relationships in E-commerce. Journal of Interactive Marketing, 38, 44-54.

Kumar, V., Aksoy, L., Donkers, B., Venkatesan, R., Wiesel, T., \& Tillmanns, S. (2010). Undervalued or overvalued customers: capturing total customer engagement value. Journal of service research, 13(3), 297-310.

Larzelere, R. E., \& Huston, T. L. (1980). The Dyadic Trust Scale: Toward Understanding Interpersonal Trust in Close Relationships. Journal of Marriage and the Family, 42(3), 595.

Leal, G. P. A., Hor-Meyll, L. F., \& de Paula Pessôa, L. A. G. (2014). Influence of virtual communities in purchasing decisions: The participants' perspective. Journal of Business Research, 67(5), 882-890.

Lin, H. C., Bruning, P. F., \& Swarna, H. (2018). Using online opinion leaders to promote the hedonic and utilitarian value of products and services. Business Horizons, 61(3), 431-442.

Liu, A. X., Steenkamp, J. B. E., \& Zhang, J. (2018). Agglomeration as a Driver of the Volume of Electronic Word of Mouth in the Restaurant Industry. Journal of Marketing Research.

Liu, A. X., Steenkamp, J. B. E., \& Zhang, J. (2018). Agglomeration as a Driver of the Volume of Electronic Word of Mouth in the Restaurant Industry. Journal of Marketing Research, 55(4), 507-523.

Lorenzo-Seva, U., Timmerman, M. E., \& Kiers, H. A. (2011). The Hull method for selecting the number of common factors. Multivariate behavioral research, 46(2), 340-364.

Luigi, D., Oana, S., Mihai, T., \& Simona, V. (2012). The use of regression analysis in marketing research. Studies in Business \& Economics, 7(2).
Malhotra, N. K., Rocha, I., Laudisio, M. C., Altheman, É., Borges, F. M., \& Taylor, R. B. (2005). Introdução à pesquisa de marketing. São Paulo: Pearson.

Martensen, A., Brockenhuus-Schack, S., \& Zahid, A. L. (2018). How citizen influencers persuade their followers. Journal of Fashion Marketing and Management: An International Journal. 22(3), 335353. https://doi.org/10.1108/JFMM-09-2017-0095.

Nhlabathi, M. P. (2016). The impact of e-word-ofmouth, engagement and brand image on soccer fans' intention to purchase football tickets (Doctoral dissertation).

Nunes, R. H., Ferreira, J. B., Freitas, A. S. D., \& Ramos, F. L. (2018). The effects of social media opinion leaders' recommendations on followers' intention to buy. Revista Brasileira de Gestão de Negócios, 20(1), 57-73.

Oliver, R. L. (1997). Effect of expectations and disconfirmation on postexposure product evaluations: an alternative interpretation. Journal of Applied Psychology, 62 (4), 480-486.

Pan, B., \& Crotts, J. C. (2012). Theoretical models of social media, marketing implications, and future research directions. Social media in travel, Tourism and hospitality: Theory, practice and cases, 73-85.

Pan, B., \& Crotts, J. C. (2012). Theoretical models of social media, marketing implications, and future research directions. Social media in travel, Tourism and hospitality: Theory, practice and cases, 1, 73-86.

Pansari, Anita \& Kumar, V (2017). Customer engagement: the construct, antecedents, and consequences. Journal of the Academy of Marketing Science, 45(3), 294-311.

Pasquali, L. (2007). Validade dos testes psicológicos: será possível reencontrar o caminho?. Psicologia: teoria e pesquisa, 23(SPE), 99107.

Pierce, J., Kostova, T. and Dirks, K. 2003. The state of psychological ownership: Integrating and extending a century of research. Review of General Psychology, 7(1), 84-107. 
Follow-Me: Análise dos Construtos que Influenciam na Tomada de Decisão de Seguidores Ligados a Influenciadores Digitais no Contexto Virtual

Raymundo, V. P. (2009). Construção e validação de instrumentos: um desafio para a psicolinguística. Letras de Hoje, 44(3), 86-93.

Rogers, E. (1983). Diffusion of Innovations (3rd ed.). New York: Free Press.

Roy Dholakia, R., \& Sternthal, B. (1977). Highly credible sources: Persuasive facilitators or persuasive liabilities?. Journal of Consumer Research, 3(4), 223232.

Sanchez-Cartas, J. M., \& Leon, G. (2018, June). On "Influencers" and Their Impact on the Diffusion of Digital Platforms. In International Conference on Practical Applications of Agents and Multi-Agent Systems (pp. 210-222). Springer, Cham.

Sashi, C. M. (2012). Customer engagement, buyerseller relationships and social media. Management Decision, 50(2), 253-272.

Schaefer, M. (2012), Return on Influence: The Revolutionary Power of Klout, Social Scoring, and Influence Marketing, McGraw-Hill, New York, NY.

Scheinbaum, A. C. (2016). Digital Engagement: Opportunities and Risks for Sponsors: ConsumerViewpoint and Practical Considerations For Marketing via Mobile and Digital Platforms. Journal of Advertising Research, 56(4), 341-345.
Shi, M., \& Wojnicki, A. (2014). Money talks... to online opinion leaders: What motivates opinion leaders to make social-network referrals? Journal of Advertising Research, 54(1), 81-91.

Sternthal, B., Dholakia, R., \& Leavitt, C. (1978). The persuasive effect of source credibility: Tests of cognitive response. Journal of Consumer research, $4(4), 252-260$.

Thakur, R. (2016). Understanding customer engagement and loyalty: a case of mobile devices for shopping. Journal of Retailing and consumer Services, 32, 151-163.

Usman, A., \& Okafor, S. (2019). Social Media and Purchase Intentions: Strategic Marketing Implications. Harnessing Omni-Channel Marketing Strategies for Fashion and Luxury Brands, 83.

Wongkitrungrueng, A., \& Assarut, N. (2018). The role of live streaming in building consumer trust and engagement with social commerce sellers. Journal of Business Research. (no prelo).

Yahia, I. B., Al-Neama, N., \& Kerbache, L. (2018). Investigating the drivers for social commerce in social media platforms: Importance of trust, social support and the platform perceived usage. Journal of Retailing and Consumer Services, 41, 11-19.

\section{Sobre os autores:}

Igor de Jesus Lobato Pompeu Gammarano - Universidade da Amazônia - UNAMA, Belém, Pará (Brasil). E-mail: igor.internacionalista@gmail.com Orcid id: https://orcid.org/0000-0001-7769-8265

Everaldo Marcelo Souza da Costa - Universidade da Amazônia - UNAMA, Belém, Pará (Brasil). E-mail: prof.emsc@gmail.com Orcid id: https://orcid.org/0000-0001-5134-4733

Sérgio Castro Gomes - Universidade da Amazônia - UNAMA, Belém, Pará (Brasil). E-mail: sergio.gomes@unama.br Orcid id: https://orcid.org/0000-0002-1731-8766

Emilio Jose Montero Arruda Filho - Universidade da Amazônia - UNAMA, Belém, Pará (Brasil). E-mail: emilio.arruda@unama.br Orcid id: https://orcid.org/0000-0002-1574-8332 


\title{
FOLLOW ME: ANALYSIS OF CONSTRUCTIONS THAT INFLUENCE ON DECISION-MAKING OF FOLLOWERS ON DIGITAL INFLUENCERS IN THE VIRTUAL CONTEXT
}

\author{
Igor de Jesus Lobato Pompeu Gammarano, Everaldo Marcelo Souza da Costa, Sérgio Castro Gomes, Emilio Jose \\ Montero Arruda Filho \\ Universidade da Amazônia - UNAMA, Belém, Pará (Brasil)
}

\section{ARTICLE DETAILS}

\section{Article history:}

Received: 1 October 2019

Accepted: 30 March 2020

Available online May: 01 th 2020

Double Blind Review System

Scientific Editor

llan Avrichir

\section{Key words}

Digital Influencers

Decision Making

Electronic Word-of-Mouth

\begin{abstract}
Objective: The present study aims to analyze the relationship between the Confidence, Electronic Mouth-to-Mouth (E-WOM) and Engagement with Consumer Decision Making constructs of individuals who follow Digital Influencers in the virtual context.

Method: The study was developed from an exploratory quantitative approach using the Survey with 147 respondents, where the exploratory factor analysis (AFE) technique was used, followed by multivariate linear regression.

Main Results: The results indicate that the Constructs Confidence and Engagement influence the Decision Making of consumption of followers of digital influencers, while Electronic Mouth-to-Mouth (E-WOM) was not significant in relation to the Decision Making of individuals who follow the digital influencers.

Relevance / Originality: Although trust, engagement, and mouth-to-mouth are widely tested constructs in consumer behavior marketing, there is still no evidence of how these constructs are effectively perceived and related in the context of digital influencers. Therefore, we seek to contribute to the literature regarding the effects of these constructs on a specific digital marketing environment, significantly new that may have an effect on the decision making process of consumers.

Theoretical / methodological contributions: The use of EFA as necessary to verify the existence of constructs in a new consumption generation environment and its association with the multivariate regression technique to determine the effects on consumers' decision making is highlighted.
\end{abstract}




\title{
SÍGUEME: ANÁLISIS DE CONSTRUCCIONES QUE INFLUYEN EN LOS INFLUYENTES DIGITALES QUE TOMAN DECISIONES EN EL CONTEXTO VIRTUAL
}

\author{
Igor de Jesus Lobato Pompeu Gammarano, Everaldo Marcelo Souza da Costa, Sérgio Castro Gomes, Emilio Jose \\ Montero Arruda Filho \\ Universidade da Amazônia - UNAMA, Belém, Pará (Brasil)
}

\section{HISTORIA DEL ARTÍCULO}

Historia del Artículo:

Recibido: 1 de Octubre de 2019

Aceptado: 30 de Marcha de 2020

Disponible en línea: 01 de Mayo 2020

Double Blind Review System

Editor Científico

Ilan Avrichir

\section{Palabras-clave:}

Influenciadores Digitales

Toma de Decisiones

Boca a Boca Electrónica

\section{RESUMEN}

Objetivo: El presente estudio tiene como objetivo analizar la relación entre la confianza, los compromisos electrónicos boca a boca (E-WOM) y la toma de decisiones del consumidor de las personas que siguen a los influenciadores digitales en el contexto virtual.

Método: El estudio se desarrolló a partir de un enfoque exploratorio cuantitativo utilizando el Survey con 147 encuestados, donde ha sido utilizado la técnica de análisis factorial exploratorio (AFE), seguida de regresión lineal multivariada.

Resultados principales: Los resultados indican que la confianza y el compromiso de los constructos influyen en la Toma de Decisiones del consumo de seguidores de personas influyentes digitales, mientras que la boca a boca electrónica (E-WOM) no ha sido significativa en relación con la toma de decisiones de las personas que Sigue a los influencers digitales.

Relevancia / Originalidad: aunque la confianza, el compromiso y el boca a boca son constructos ampliamente probados en el marketing de comportamiento del consumidor, todavía no hay evidencia de cómo estos constructos se perciben y relacionan de manera efectiva en el contexto de los influencers digitales. Por lo tanto, la búsqueda es contribuir a la literatura sobre los efectos de estas construcciones en un entorno de marketing digital específico, significativamente nuevo que puede tener un efecto en el proceso de toma de decisiones de los consumidores.

Contribuciones teóricas / metodológicas: se ha destacado el uso de EFA según sea necesario para verificar la existencia de construcciones en un nuevo entorno de generación de consumo y su asociación con la técnica de regresión multivariante para determinar los efectos en la Toma de Decisiones de los consumidores.

Para citar este artigo:

Gammarano, I., Souza da Costa, E., Gomes, S., \& Arruda Filho, E. (2020). Follow-Me: Análise dos Construtos que Influenciam na Tomada de Decisão de Seguidores Ligados a Influenciadores Digitais no Contexto Virtual. Internext, 15(2), 90-106. doi:http://dx.doi.org/10.18568/internext.v15i2.582 